\title{
Efeitos hemodinâmicos e metabólicos iniciais da perfusão hipotérmica intestinal in situ. Avaliação de um novo modelo canino de autotransplante intestinal $^{1}$
}

\author{
Initial hemodynamic and metabolic effects of intestinal hypothermic perfusion
} in situ. An alternative model of canine intestinal autotransplantation.

\author{
Ruy Jorge Cruz Junior ${ }^{2}$, Cristiano de Jesus Correia ${ }^{3}$, Luiz Francisco Poli de \\ Figueiredo ${ }^{4}$, Maurício Rocha e Silva ${ }^{5}$
}
1. Estudo realizado no Serviço de Fisiologia Aplicada - Instituto do Coração (InCor) da Faculdade de Medicina da Universidade de São Paulo (FMUSP) e Laboratório de Investigações Médicas (LIM -11) da FMUSP.
2. Médico-assistente - Doutor, do InCor da FMUSP.
3. Pesquisador do Laboratório de Investigações Médicas (LIM -11) da FMUSP.
4. Professor Associado do Depto. de Cardiopneumologia da FMUSP.
5. Professor Titular do Depto. de Cardiopneumologia da FMUSP.

\section{RESUMO}

O transplante intestinal tem sido utilizado como uma alternativa promissora no tratamento da síndrome do intestino curto em pacientes adultos e pediátricos. Vários modelos experimentais foram desenvolvidos com a finalidade de testar diferentes soluções de preservação. No entanto são modelos complexos que levam, muitas vezes, um tempo prolongado para a sua realização.

OBJETIVO: Neste estudo, desenvolvemos um modelo alternativo de autotransplante intestinal in situ analisando os efeitos hemodinâmicos e metabólicos iniciais da perfusão hipotérmica com solução de Ringer lactato.

MÉTODOS : Foram utilizados seis cães machos sem raça definida $(22,8 \pm 1,4 \mathrm{Kg})$; as variáveis hemodinâmicas sistêmicas foram obtidas por meio de cateter arterial e pelo SwanGanz. A perfusão do trato gastrintestinal foi avaliada pela medida do fluxo sangüíneo da veia mesentérica superior (FSVMS, fluxômetro ultra-sônico), e através da medida do $\mathrm{pCO}_{2}$ intestinal ( $\mathrm{pCO}_{2}$-int e $\mathrm{pCO}_{2}$-gap, tonometria a gás). Inicialmente realizamos a secção do jejuno proximal e íleo distal e isolamento dos vasos mesentéricos com fitas cardíacas, todo o tecido nervoso e linfático em torno do eixo vascular intestinal foi seccionado. $\mathrm{O}$ território mesentérico foi perfundido através da artéria mesentérica superior por 30 minutos com 1000 $\mathrm{ml}$ de Ringer lactato a $4^{0} \mathrm{C}$, e o efluente drenado através de uma pequena incisão na veia mesentérica superior. Os animais foram observados por $120 \mathrm{~min}$ após o inicio do período de reperfusão. Amostras de sangue foram obtidas da aorta abdominal, para análise gasométrica. RESULTADOS : A perfusão intestinal hipotérmica induziu uma redução do FSMVS apenas nos primeiros 30 min de reperfusão $(587 ? 70,9$ para $398 ? 102,8 \mathrm{ml} / \mathrm{min}$ ) e um aumento do pCO2-gap (2?2,7 para 29,8?6 $\mathrm{mmHg})$. Não foram observadas alterações significativas em relação a parâmetros hemodinâmicos e metabólicos sistêmicos (PAM, DC, pH, excesso de bases, hemoglobina) assim como na temperatura central.

CONCLUSÃ̃O: O modelo de autotransplante intestinal é extremamente útil e de fácil execução, para a avaliação inicial de soluções de preservação e/ou drogas antioxidantes, comumente utilizadas no transplante de intestino. 
Descritores: Transplante intestinal. Tonometria a gás. Perfusão hipotérmica. Soluções de preservação.

\begin{abstract}
Intestinal transplantation is an acceptable therapy for children and adults with short bowel syndrome. The great majority of large animal experimental models of intestinal transplantation are complex and take a lot of time to be performed. In this study, we developed an alternative model of intestinal autotransplantation and evaluate the initial impact of isolated hypothermic intestinal perfusion with Ringer's lactate solution on hemodynamic and metabolic parameters.
\end{abstract}

METHODS: Six pentobarbital anesthetized mongrel dogs were used in this study $(22,8 \pm 1,4$ $\mathrm{Kg}$ ). Systemic hemodynamic were evaluated through a Swan-Ganz and arterial catheters; while gastrointestinal tract perfusion by superior mesenteric vein blood flow (SMVBF, ultrasonic flowprobe) and intestinal mucosal $\mathrm{pCO}_{2}\left(\mathrm{pCO}_{2}\right.$-int and $\mathrm{pCO}_{2}$-gap, gas tonometry). Initially, the proximal jejunum and distal ileum were transected; at the basis of the mesentery excepting the superior mesenteric artery and vein. The small bowel was then perfused in situ with cold $\left(4^{0} \mathrm{C}\right)$ Ringer's lactate solution for 30 minutes, with an automatic pump. The animals where observed for 120 minutes after reperfusion. Blood samples were collected from thoracic aorta for gas blood analysis.

RESULTS : Hypothermic intestinal perfusion induced a partial reduction on SMVBF, only in the first $30 \mathrm{~min}$ of reperfusion (398? 102,8 to $587 ? 70,9 \mathrm{ml} / \mathrm{min}$ ) and an increase on pCO2-gap $(2 ? 2,7$ to $29,8 ? 6 \mathrm{mmHg})$. During the experimental protocol, we did not observe significant alterations on systemic hemodynamic or metabolic parameters (MAP, $\mathrm{CO}, \mathrm{pH}$, base excess and hemoglobin levels) as well as on central core temperature.

CONCLUSION: The model of intestinal transplantation is very useful to test different preservation solutions and/or antioxidants drugs, which are often used in clinical small bowel transplantation.

Key words : Small bowel transplantation. Gas tonometry. Hypothermic perfusion.

\title{
Introdução
}

O transplante intestinal tem sido utilizado como uma alternativa promissora no tratamento da síndrome do intestino curto em pacientes adultos e pediátricos. Apesar de uma elevada taxa de complicações, a sobrevida de cinco anos do enxerto intestinal varia de 50 a $70 \%$ em muitos centros americanos. ${ }^{1-3}$ Esse aumento da sobrevida do enxerto deve-se principalmente à utilização de novas drogas imunossupressoras e a realização do transplante combinado fígadointestino.

Além do controle da rejeição, o sucesso do transplante intestinal está relacionado à minimização da lesão oxidativa após o fenômeno de isquemia e reperfusão mesentérica. ${ }^{4}$ Neste ínterim, várias soluções de preservação foram utilizadas com a finalidade de aumentar o tempo de preservação do órgão e reduzir os efeitos deletérios da isquemia hipotérmica, podendo desta forma ter impacto fundamental na viabilidade do enxerto a curto e médio prazo.

Desta forma vários modelos experimentais em animais de grande e pequeno porte foram desenvolvidos, com o objetivo de estudar as diferentes soluções de preservação disponíveis, assim como o efeito final da denervação do território mesentérico. ${ }^{4-11}$ 
Com a finalidade de analisarmos o efeito isolado da perfusão hipotérmica no território intestinal e sua posterior reperfusão, desenvolvemos um modelo alternativo de autotransplante intestinal in situ em cães analisando os efeitos hemodinâmicos e metabólicos iniciais da perfusão hipotérmica com solução de Ringer lactato.

\section{Métodos}

Este projeto foi realizado conforme com as normas internacionais para utilização de animais de experimentação.

\section{Preparação animal}

Foram utilizados seis cães sem raça definida, com peso de $22,8 \pm 1,4 \mathrm{~kg}$. Os animais foram submetidos a um período de jejum de 12 horas, porém com livre acesso a água. A anestesia foi realizada com injeção intravenosa de pentobarbital sódico, $25 \mathrm{mg} / \mathrm{Kg}$; sendo os animais posicionados em decúbito dorsal sob colchão térmico. Após a entubação orotraqueal, os cães foram submetidos à ventilação mecânica. $\mathrm{O}$ respirador foi ajustado com volume corrente de $15 \mathrm{ml} / \mathrm{Kg}, 1,0$ de fração inspirada de oxigênio e frequiência respiratória de $14 \mathrm{mpm}$. Doses adicionais de pentobarbital foram utilizadas quando necessárias.

Através de uma inguinotomia direita, foi realizada a cateterização da veia femoral, sendo posicionado um cateter de polietelieno (PE240), no interior da veia cava inferior. Após a obtenção do acesso venoso, foi aplicado $0,5 \mathrm{mg}$ de atropina. Um outro cateter de polietileno foi introduzido na artéria carótida comum direita, para medida da pressão arterial média e colheita de sangue arterial para análise gasométrica, $\mathrm{pH}$, bicarbonato, déficit de bases, hematócrito e hemoglobina.

Através da veia jugular externa direita, foi introduzido cateter de Swan-Ganz? 93A-131H-7F (Baxter Health Care Corporation, Irvine, CA - EUA), cuja extremidade distal foi posicionada na artéria pulmonar, por meio da análise das curvas de pressão. Este cateter foi conectado ao monitor de débito cardíaco (Vigilance, Baxter Edwards Critical Care, Irvine, CA - EUA), permitindo a medida da pressão média da artéria pulmonar (PMAP), débito cardíaco (DC) e temperatura central.

As medidas pressóricas foram realizadas através da conexão dos respectivos cateteres a transdutores de pressão (Transpac Disposable Transducer, Abbott, Chicago, IL - EUA) ligados em um sistema de aquisição de dados biológicos (modelo MP 100, Biopac System Inc., Goleta, CA - EUA) sendo os dados registrados em um computador através de software específico (ACqKnowledge? IIII MP 100 WSW).

\section{Modelo de auto-transplante intestinal}

1. O acesso à cavidade abdominal foi obtido por incisão mediana.

2. Inicialmente, realizamos, a seccção do jejuno proximal a $10 \mathrm{~cm}$ do ângulo de Treitz, sendo realizada a ligadura de todo o mesentério em direção ao eixo vascular. O mesmo procedimento foi realizado no íleo terminal a aproximadamente $10 \mathrm{~cm}$ da válvula ileocecal (o fechamento das extremidades intestinais foi realizada com sutura contínua, com algodão 2.0);

3. em seguida, foi realizada a dissecção da artéria e veia mesentérica em sua porção mais proximal, sendo estes vasos isolados e reparados com fitas cardíacas. Um fluxômetro ultra-sônico foi posicionado ao redor da veia mesentérica (T206 small animal blood flowmeter - Transonic Systems Inc., Nova York - EUA) para a medida contínua do fluxo sangüíneo neste vaso (FSVMS). (Figura 1A). 
4. Uma incisão de aproximadamente $0,5 \mathrm{~cm}$ foi realizada na borda contra-mesentérica do íleo (aproximadamente $20 \mathrm{~cm}$ do coto distal) sendo introduzido, em direção proximal, uma sonda de tonometria gástrica (cateter TRIP? Tonometric Catheter - 16F / Tonometrics Division, Helsinque - Finlândia). Este cateter foi conectado ao sistema de leitura TONOCAP? (Datex-Engstrom Division, Helsinki - Finlândida), permitindo a medida da pressão parcial de dióxido de carbono da mucosa intestinal $\left(\mathrm{pCO}_{2}\right.$-int); as alças intestinais foram protegidas com compressa úmida em soro fisiológico, sendo então realizada a oclusão da artéria e veia mesentérica superior com pinças vasculares não traumáticas.

5. Através de uma abertura transversal na parede anterior da artéria mesentérica superior, foi introduzida uma sonda de Levine para a realização da perfusão hipotérmica do intestino delgado com solução de Ringer lactato a $4^{\circ} \mathrm{C}$, por 30 minutos (taxa de infusão $33,3 \mathrm{ml} / \mathrm{min}$ ), uma outra pequena incisão foi realizada na veia mesentérica superior para drenagem da solução de perfusão (Figuras 1B e 1C).

6. Após a perfusão hipotérmica realizamos a retirada da sonda de Levine e fechamento da arteriotomia e venotomia com fio de polipropileno 6-0. 

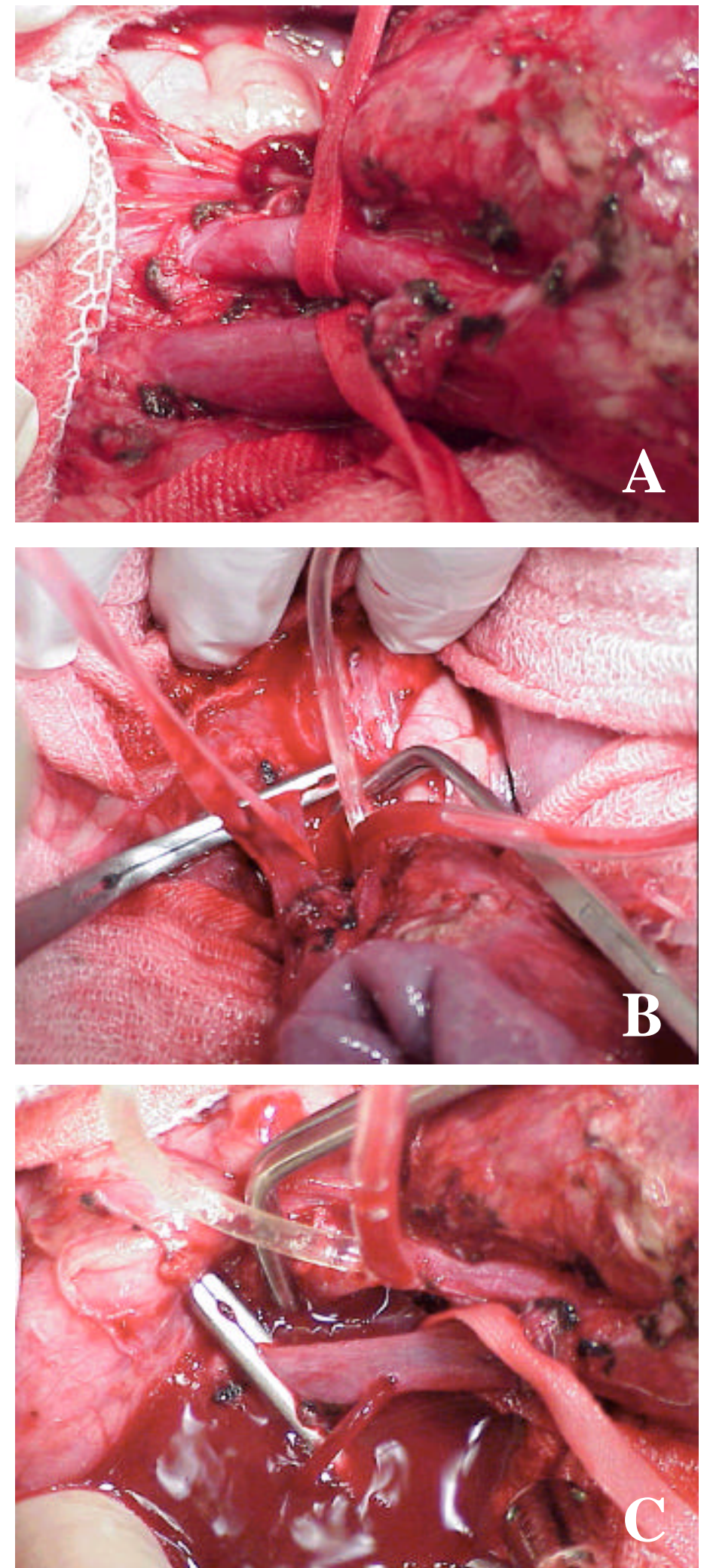

FIGURA 1 - Modelo alternativo de autotransplante intestinal: (A) dissecção e isolamento completo da artéria e veia mesentérica superior; (B) pinçamento dos vasos mesentéricos e posicionamento do cateter de polietileno no interior da artéria mesentérica superior, para a realização da perfusão hipotérmica do intestino delgado; (C) drenagem da solução de perfusão através de uma pequena incisão realizada na parede anterior da veia mesentérica superior. 


\section{Protocolo experimental}

Trinta minutos após o final da preparação, foram obtidas as medidas basais (BL). Em seguida foi realizada a oclusão dos vasos mesentéricos, sendo iniciada a perfusão do intestino delgado com $1000 \mathrm{ml}$ de solução de Ringer lactato a $4^{\circ} \mathrm{C}$, por 30 minutos, com o auxílio de bomba automática (Harvard Apparatus, Massachusetts - EUA), conforme descrito anteriormente. O cateter da artéria mesentérica foi retirado sendo realizada a sutura da artéria e da veia mesentérica superior. Após 45 minutos de isquemia intestinal foram realizadas novas medidas hemodinâmicas (II45).

Após, a retirada das pinças vasculares, os animais foram observados por um período adicional de duas horas (R0 a R120). Ao final do experimento os animais foram sacrificados com infusão de pentobarbital sódico $(50 \mathrm{mg} / \mathrm{Kg})$, seguido de infusão de $20 \mathrm{ml}$ de cloreto de potássio hipertônico.

\section{Variáveis analisadas}

A pressão arterial média, temperatura central e o fluxo sangüíneo da veia mesentérica superior foram avaliados continuamente. O débito cardíaco foi determinado pela técnica de termodiluição, utilizando injeção em bolus de $3 \mathrm{ml}$ de solução salina isotônica a $20^{\circ} \mathrm{C}$, a cada 10 minutos.

$\mathrm{O}$ déficit de bases, $\mathrm{pH}, \mathrm{pCO} 2, \mathrm{pO}_{2}$, saturação de hemoglobina, hematócrito, e o bicarbonato nas amostras de sangue proveniente da aorta abdominal foram obtidos nos momentos basais (BL) e 30, 60 e 120 minutos após o início da reperfusão (R30, R60 e R120, respectivamente). As amostras de sangue foram analisadas imediatamente após a colheita pelo analisador de gases Stat Profile Ultra Analyzer (Nova Biomedical, Waltham, MA, EUA).

\section{Tonometria intestinal}

$\mathrm{O}$ pCO2 da mucosa intestinal (pCO2-int) foi avaliado automaticamente a cada 10 minutos, através da tonometria a gás. $\mathrm{O}$ pCO2-gap foi calculado através da diferença entre a $\mathrm{pCO}_{2}$ da mucosa intestinal e a $\mathrm{pCO}_{2}$ arterial.

\section{Metodologia estatística}

Os resultados são apresentados como média ? erro padrão. Análise estatística foi realizada usando o software Statistic Package for Social Sciences for Windows (versão 6,0 - SPSS Inc., Chicago, IL, EUA). Foi utilizada análise de variância controlada pelo teste de Tukey. Diferença estatística foi considerada para valores de $p$ menores que 0,05 .

\section{Resultados}

Todos os animais apresentavam-se hemodinamicamente estáveis no início do experimento. No final do período de perfusão hipotérmica, não foram observadas alterações significativas em relação as variáveis hemodinâmicas avaliadas (pressão arterial média e pressão média da artéria pulmonar, débito cardíaco), exceto a ausência de fluxo sangüíneo mesentérico decorrente da oclusão dos vasos mesentéricos (Tabela 1). Neste período, pudemos observar um aumento progressivo do $\mathrm{pCO}_{2}$-int $\left(37 ? 4,3\right.$ para 65,3 ? 7,6 $\mathrm{mmHg}$ ) e do $\mathrm{pCO}_{2}$-gap $(2$ ? 2,7 para 29,8? $6 \mathrm{mmHg})(p<0,05)$.

No período de reperfusão intestinal não ocorreram alterações significativas dos parâmetros hemodinâmicos avaliados (PAM, PMAP e DC). Observamos, no entanto, uma recuperação 
parcial do fluxo sangüíneo da veia mesentérica superior durante a fase inicial de reperfusão, (398? 102,8 $\mathrm{ml} / \mathrm{min} v s$. $587 ? 70,9 \mathrm{ml} / \mathrm{min})$. O restabelecimento do fluxo para o intestino delgado proporcionou uma redução progressiva, porém não significativa, do $\mathrm{pCO}_{2}$-int e do $\mathrm{pCO}_{2}$-gap mesmo após duas horas do início da reperfusão (Tabela 1). Apesar da utilização da perfusão hipotérmica in situ, não foi observada redução significativa da temperatura central. Não ocorreram alterações significativas do $\mathrm{pH}$ e da hemoglobina arterial durante todo o protocolo experimental (Tabela 1).

TABELA 1 - Pressão arterial e da artéria pulmonar média (PAM, PMAP), débito cardíaco (DC) fluxo sangüíneo da veia mesentérica superior (FSVMS) pCO2-int, pCO2-gap, temperatura central, $\mathrm{pH}$ e hemoglobina arterial durante o momento basal (BL), no final do período de isquemia hipotérmica (II45), e 30, 60 e 120 minutos após o início da reperfusão (R30, R60 e R120).

\begin{tabular}{|c|c|c|c|c|c|}
\hline & BL & II45 & 1. $\mathrm{R} 30$ & R60 & R120 \\
\hline $\operatorname{PAM}(m m H g)$ & $125 ? 8.6$ & $129 ? 7.1$ & $115 ? 9.8$ & $112 ? 10.1$ & $115 ? 8.9$ \\
\hline $\operatorname{PMAP}(m m H g)$ & $16.2 ? 1.5$ & $15.6 ? 1.0$ & $16.1 ? 1.3$ & $15.8 ? 1.4$ & $15.8 ? 1.3$ \\
\hline $\mathrm{DC}(L / \mathrm{min})$ & $3.2 ? 0.3$ & $3.1 ? 0.1$ & $2.5 ? 0.3$ & $2.6 ? 0.4$ & $2.5 ? 0.4$ \\
\hline FSVMS ( $\mathrm{ml} / \mathrm{min})$ & $587 ? 70.9$ & $0^{a}$ & $398 ? 102,8^{a}$ & $421 ? 73.2$ & $405,6 ? 102.8$ \\
\hline pCO2-int $(m m H g)$ & $37 ? 4.3$ & $73 ? 3.6^{a}$ & $65.3 ? 7.6^{a}$ & $64 ? 11.8^{a}$ & $52.8 ? 7.6^{a}$ \\
\hline pCO2-gap $(m m H g)$ & $2 ? 2.7$ & - & $29.8 ? 6^{a}$ & $30.6 ? 9.8^{a}$ & $21.7 ? 7.4^{a}$ \\
\hline temperatura $\left({ }^{0} C\right)$ & $36.1 ? 0.1$ & $36.4 ? 0.2$ & $36.3 ? 0.2$ & $36.1 ? 0.4$ & $36.8 ? 0.5$ \\
\hline $\mathrm{pH}$ arterial & $7.37 ? 0.01$ & - & $7.33 ? 0.02$ & $7.35 ? 0.02$ & $7.35 ? 0.02$ \\
\hline $\mathrm{Hb}$ - arterial $(g / d l)$ & $11.3 ? 1.2$ & - & $12.8 ? 1.1$ & $12.1 ? 1.2$ & $12.1 ? 1.5$ \\
\hline
\end{tabular}

Legendas: ${ }^{a} p<0,05 v s . \mathrm{BL}$

\section{Discussão}

Vários são os modelos utilizados com o objetivo de avaliar os efeitos imediatos e tardios do transplante de intestino em animais de grande porte. Os modelos de autotransplante intestinal mimetizam as alterações decorrentes do alotransplante, mas eliminam, por razões óbvias, os efeitos decorrentes da ativação do sistema imunológico. Geralmente, o procedimento consiste na retirada do órgão da cavidade, perfusão e preservação do órgão com soluções e temperaturas adequadas, e finalmente, o reimplante do intestino em seu lugar de origem. No entanto, são modelos complexos que levam muitas vezes, um tempo cirúrgico prolongado, além da necessidade de pessoal treinado para a realização anastomoses vasculares. Além disso, muitos grupos apresentaram taxas de mortalidade extremamente altas, de até $50 \%$, com o uso deste modelo. ${ }^{4,6,8,9}$ 
Por razões econômicas e práticas, outros grupos realizam apenas a secção de todo o tecido linfático e nervoso na raiz do eixo mesentérico, sem a indução de isquemia normo ou hipotérmica. ${ }^{10,12}$ Estes modelos são utilizados para a avaliação do trânsito e da capacidade de absorção intestinal. No entanto, a lesão de isquemia e reperfusão, não é considerada e/ou avaliada neste tipo de experimento.

Portanto os modelos de autotransplante apresentam dois objetivos distintos para a sua realização: (1) a avaliação isolada dos efeitos da isquemia e reperfusão intestinal e (2) estudo dos efeitos da denervação do território mesentérico.

Neste estudo, desenvolvemos um modelo alternativo de autotransplante intestinal de fácil e rápida execução, analisando os efeitos hemodinâmicos e metabólicos iniciais da perfusão hipotérmica com solução de Ringer lactato, sem a retirada do órgão da cavidade. Este modelo permite avaliar além dos efeitos da perfusão hipotérmica, as alterações decorrentes da denervação mesentérica.

No modelo apresentado, não observamos o desenvolvimento de hipotermia mesmo com a realização da perfusão hipotérmica intestinal in situ; além disso, os valores de hemoglobina se mantiveram em torno de $12 \mathrm{~g} / \mathrm{dl}$ durante todo o experimento, denotando um pequeno sangramento durante a fase de perfusão do território mesentérico. Outros achados importantes foram à ausência de alterações hemodinâmicas significativas e desenvolvimento de acidose metabólica durante a fase reperfusão.

Pudemos observar, neste estudo, a susceptibilidade da camada mucosa intestinal ao fenômeno de isquemia e reperfusão, através da elevação dos valores da $\mathrm{pCO}_{2}$-int e do $\mathrm{pCO}_{2}$-gap, mesmo com a utilização perfusão hipotérmica com Ringer lactato. Acreditamos, no entanto, que a hipotermia promoveu um fator protetor à camada mucosa durante a fase de reperfusão, visto que em estudos previamente realizados pelo nosso grupo, utilizando modelos de isquemia normotérmica através da oclusão da aorta e exclusão hepática, a elevação da $\mathrm{pCO}_{2}$ na mucosa do trato digestivo foi significativamente maior e sua recuperação ulterior, denotando um processo de isquemia da camada mucosa muito mais acentuado. ${ }^{13,14}$

Vários são os estudos que correlacionam as alterações histopatológicas com as variações da $\mathrm{pCO}_{2}$ da mucosa do intestino. ${ }^{15,16}$ Desta forma a tonometria intestinal ou mesmo gástrica, pode ser utilizada como um marcador importante na detecção da inviabilidade do enxerto ou mesmo da lesão de isquemia e reperfusão na qual o intestino é submetido, conforme demonstrado em diversos estudos clínicos e experimentais.

Nosso estudo apresenta algumas limitações, como curto período de isquemia hipotérmica o qual o enxerto é submetido, além da não perfusão hipotérmica do lúmen intestinal; fatores estes que podem ser facilmente modificados sem prejuízos ao modelo em estudos futuros. Além disso, um tempo maior de observação após a reperfusão seria necessário para estabelecer em que momento é restaurado o fluxo sangüíneo mesentérico assim como a perfusão da mucosa intestinal.

\section{Conclusão}

O modelo de autotransplante intestinal é extremamente útil e de fácil execução, para a avaliação inicial de soluções de preservação e/ou drogas antioxidantes, comumente utilizadas no transplante de intestino.

\section{Referências}


1. Abu-Elmagd K, Reyes J, Todo S, Rao A, Lee R, Irish W, Furukawa H, Bueno J, McMichael J, Fawzy AT, Murase N, Demetris J, Rakela J, Fung JJ, Starzl TE. Clinical intestinal transplantation: new perspectives and imunologic considerations. J Am Coll Surg 1998; 186(5): 512-25.

2. Abu-Elmagd KM, Reyes J, Fung JJ, Mazariegos G, Bueno J, Janov C, Colangelo J, Rao A, Demetris A, Starzl TE. Evolution of clinical intestinal transplantation: improved outcome and cost effectiveness. Transplant Proc 1999; 31(1-2):582-4.

3. Cicalese L, Baum C, Brown M, Sileri P, Smith D, Abcarian H, Benedetti E. Segmental small bowel transplant from adult living-related donors. Transplant Proc 2001; 33:1553.

4. Niebel W, Beglinger C, Singer MV. Hormonal control of pancreatic secretion by intrajejunal $\mathrm{HCl}$ : studies in dogs with an autotransplanted entire jejunoileum. Pancreas 1991; 6(3):341-9.

5. Newell KA, Fishbein TM. Experimental models of small bowel transplantation. Curr Op Org Transp 2003; 8:209-16.

6. Ferencz A, Szanto Z, Borsiczky B, Kiss K, Kalmar-Nagy K, Szeberenyi J, Horvath PO, Roth E. The effects of preconditioning on the oxidative stress in small-bowel autotransplantation. Surgery 2002;132(5):877-84.

7. Mueller AR, Platz KP, Heckert C, Hausler M, Schuppan D, Lobeck H, Neuhaus P. Differentiation between preservation reperfusion injury and acute rejection after small bowel transplantation. Transplant Proc 1998;30:2657-9.

8. Yagmurdur MC, Ozdemir A, Coskun T, Kilinç K, Ozenç A. Effects of ? -tocopherol on reperfusion injury in the canine small bowel autotransplantation model. Transplant Proc 1998;30:824-7.

9. Takeyoshi I, Zhang S, Nomoto M, Zhu Y, Kokudo Y, Suzuki T, Hamada N, Nemoto A, Starzl TE, Todo S. Mucosal damage and recovery of the intestine after prolonged preservation and transplantation in dogs. Transplantation 2001;71(1):1-7.

10. Oishi AJ, Sarr MG. Intestinal transplantation: effects on ileal enteric absorptive physiology. Surgery 1995;117:545-53.

11. Lopez F, Hernandez G, Castillo L, Rivera C, Añazco R, Leiva G, Zuñiga A. Usefulness of intraluminal tonometry as a method for assessment of graft ischemia after small bowel transplantation. Transplant Proc 1996; 28(5):2648-9.

12. Sarr MG, Duenes JA, Walters AM. Jejunal and ileal absorptive function after a model of canine intestinal autotransplantation. J Surg Res 1991, 51:233-9.

13. Cruz Junior RJ, Poli de Figueiredo LF, Braz JLM, Diniz EP, Rocha e Silva M. Systemic and regional effects of supraceliac aortic occlusion during hepatic vascular exclusion. Am J Surg 2003;185:388-93.

14. Cruz Junior RJ, Poli de Figueiredo LF, Braz JLM, Rocha e Silva M. Effects of intra-aortic balloon occlusion on intestinal perfusion, oxygen metabolism and gastric mucosal PCO2 during experimental hemorrhagic shock. Eur Surg Res 2004 (in press).

15. Poli de Figueiredo LF, Silva E, Cruz Junior RJ, Rocha e Silva M. Gas tonometry for evaluation of gastrointestinal mucosal perfusion: Experimental models of trauma, shock and complex surgical maneuvers - Part 1. Acta Cir Bras 2002;17(4): 211-9.

16. Iwanami K, Takeyoshi I, Ohwada S, Kobayashi J, Kawata K, Matsumoto K, Morishita Y. Intramucosal $\mathrm{pH}$ and intestinal mucosal damage in ischemia-reperfusion injury. Transpl Int 1998;11:401-7.

Correspondência:

Ruy Jorge Cruz Junior

Rua Marivaldo Fernandes, 140

04792-060 São Paulo - SP

Tel/Fax: (11)3085-7887

expcruzjr@incor.usp.br

Recebimento: 15/04/2004

Revisão: 21/05/2004

Aprovação: 18/06/2004

Conflito de interesse: nenhum

Fonte de financiamento: FAPESP (98/15658-0)

\section{Como citar este artigo:}

Cruz Jr RJ, Correia CJ, Poli de Figueiredo LF, Rocha e Silva M. Efeitos hemodinâmicos e metabólicos iniciais da perfusão hipotérmica intestinal in situ : avaliação de um novo modelo canino de autotransplante intestinal. Acta Cir Bras [serial online] 2004 Jul-Ag;19(4). Disponível em URL: http://www.scielo.br/acb [também em CDROM]. 
